\title{
Graphdiynes interacting with metal surfaces: first-principles electronic and vibrational properties
}

\author{
Simona Achilli ${ }^{a, b, c}$, Alberto Milani ${ }^{d}$, Guido Fratesi ${ }^{a, b, c}$, Francesco \\ Tumino $^{d}$, Nicola Manini ${ }^{a, b}$, Giovanni Onida ${ }^{a, b, c}$, Carlo S. \\ Casari $^{d, *}$ \\ ${ }^{a}$ Dipartimento di Fisica "Aldo Pontremoli", ${ }^{b}$ European Theoretical Spectroscopy \\ Facility (ETSF). ${ }^{c}$ INFN, Sezione di Milano, I-20133 Milano, Italy. ${ }^{d}$ Micro and \\ Nanostructured Material Lab - NanoLab, Dipartimento di Energia, Politecnico di \\ Milano, Via Ponzio 34/3 I-20133 Milano, Italy \\ E-mail: *carlo.casari@polimi.it
}

\begin{abstract}
Graphdiynes (GDYs) represent a class of 2D carbon materials based on

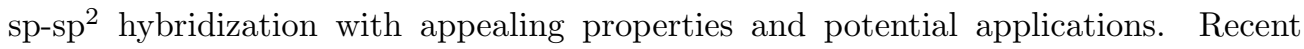
advances have demonstrated the experimental self-assembly of GDYs on metal substrates. Here we focus on $\alpha$ - and $\beta$-GDYs on $\mathrm{Au}(111)$ and $\mathrm{Pt}(111)$, and investigate how their electronic and vibrational properties are affected by the interaction with a metal substrate. We adopt hydrogenated GDY, previously characterized experimentally, as a benchmark for density functional theory simulations, that we apply to show that $\mathrm{Au}$ and $\mathrm{Pt}$ substrates impose a different degree of distortion on both $\alpha$ - and $\beta$-GDY. By comparing the adsorbed and the freestanding structures, we evaluate the effect of the surface interaction on the bandstructure and the simulated Raman spectra. Different charge transfers result in different energy shift of the Dirac cone in semi-metallic $\alpha$-GDY and changes from semiconducting to metallic behavior for $\beta$-GDY. These changes in electronic properties are accompanied by characteristic frequency shifts and modifications of Raman active modes. Our results contribute in the understanding of the metal-interaction effects on GDYs and can open a route to the design of novel 2D materials with tailored properties.
\end{abstract}

Keywords: Graphdiynes, DFT, Raman, sp-carbon, 2D carbon structures

\section{Introduction}

Graphdiynes (GDYs) are receiving increasing interest as a new class of 2D carbon materials [1, 2, 3, 4, 5]. Different GDY structures can be conceived by playing with $\mathrm{sp}^{2}$ and sp hybridized carbon atoms [6, 7, 8, 9]. For instance, starting from graphene and conceptually substituting $\mathrm{sp}^{2}$ carbon atoms with diacetylenic linear units as linkages, a total of 26 possible novel 2D carbon crystals (with up to 8 atoms per unit cell) 
can be formed with variable $\mathrm{sp} / \mathrm{sp}^{2}$ ratio, porosity and density [10]. Such 2D materials exhibit electronic properties ranging from metallic to semi-metallic with Dirac cones and to semiconducting with different energy gap, making them appealing for technological applications [11, 12, 13, 14, 15]. The experimental realization of GDY is quite recent and it is mainly related to the so-called $\gamma$-GDY showing interesting performances for photocatalysis, catalysis, photovoltaics and electronic device realization [16, 11, 17]. In this framework, Raman spectroscopy is one of the key techniques for the characterization of nanostructured carbon materials, including GDY-based systems [18, 19, 20]. For its particular sensitivity to the local molecular bond, hybridization state, and to the structural order, Raman spectroscopy is currently a fundamental tool to recognize the fingerprints of specific sp-carbon based materials with respect to other carbon nanostructures [21].

The synthesis approach for GDYs is mainly based on the use of reactive molecular building blocks which assemble in a 2D structure, usually catalyzed by the presence of a metal [22]. Such approach, first developed in the liquid phase, can also be used in vacuum exploiting on-surface synthesis techniques. When the on-surface synthesis is performed in ultra high vacuum on atomically flat metal surfaces (e.g., $\mathrm{Au}(111), \mathrm{Cu}(111), \mathrm{Pt}(111))$, a direct atomic scale characterization by means of surface science techniques such as scanning tunneling microscopy (STM) becomes possible [23, 24, 25, 26, 27]. Different works used STM to study on-surface synthesized sp-sp ${ }^{2}$ carbon structures with impressive imaging capabilities, showing the formation process down to the atomic scale and visualizing the carbon-carbon bonds [28].

The specific metal substrate suitable for GDY synthesis (e.g., copper, gold, platinum, silver) plays a complex role in modifying the carbon overlayer properties through different phenomena, including strain to adapt to the metal surface lattice parameters, charge transfer, and even partial hybridization of carbon atoms with the metal substrate ones. For instance, the $\gamma$-GDY metal contact has been recently theoretically investigated in view of a field effect transistor realization [29].

The case of hydrogenated GDY systems (h-GDYs) has been studied by means of a combined Raman spectroscopy and STM investigation [30]. The theoretically computed electronic and vibrational properties for an $\mathrm{Au}(111)$-synthesized h-GDY system turned out to be consistent with its experimentally measured properties. In addition, the theoretical analysis naturally allows one to compare the surface-deposited case with the freestanding GDY, outlining the importance of interaction effects with the metal surface. Even though recent works have shown the possibility to exfoliate GDY from multilayer samples and to transfer flakes to insulating substrates, [31] the presence of a metal substrate is usually required in the synthesis step so that the majority of GDY systems experimentally investigated so far is interacting with or supported on a metal.

Hence, we focus here on the so-far poorly explored possibilities provided by metal substrates to tune the GDY properties as an additional versatility for material engineering. In this work, we analyze two different GDY structures on two typical metals, namely $\alpha$-GDY and $\beta$-GDY on $\mathrm{Au}(111)$ and $\operatorname{Pt}(111)$, as representative 
substrates capable of a weak and relatively strong interaction with GDYs, respectively. The freestanding $\alpha$-GDY and $\beta$-GDY display dissimilar electronic properties: $\alpha$-GDY is a semimetal, with a Dirac cone at the Fermi level and graphene-like band structure, while $\beta$-GDY is a semiconductor with a direct gap of $0.26 \mathrm{eV}$, obtained in the present work by means of density-functional theory (DFT) with the Perdew-Burke-Ernzerhof (PBE) exchange-correlation functional. $\alpha$ - and $\beta$-GDYs and their interaction with the supporting metal surfaces are poorly investigated in the literature which focuses mainly on the $\gamma$-GDY [17, 19] and on similar available systems, also studied in recent experimental works [32, 30, 33, 34].

Our theoretical analysis focuses on the effects of the interaction between GDY and the underlying metals, in term of structural deformation, charge transfer, and changes in the electronic band structure. The study includes a detailed analysis of the consequent effects on the GDY's vibrational properties. In particular, we investigate how the overlayer-substrate interaction modifies the electronic properties at the Fermi level, with particular attention to the changes induced on the Dirac cone in the semimetallic $\alpha$-GDY case, and to the possibility to turn the semiconductor $\beta$-GDY into a metallic system. Due to the changes in the electronic structure, and in view of the strong electron-phonon coupling present in these materials [9, 18], substantial effects are to be expected in the Raman spectra. Indeed, these spectra turn out to be strongly sensitive to the interaction with the substrate, and can be used to monitor and to predict the GDY structural changes due to the interaction with the metal surface.

On the other hand, vibrational properties, and in particular the Raman response, of these sp-sp ${ }^{2}$ hybrid materials have been only rarely investigated by DFT-based theoretical works. It is indeed often computationally prohibitive to deal with extended systems characterized by a large periodicity imposed by the matching between the carbon network and the substrate, in the presence of metallicity. For this reason, simplified models are commonly adopted in such simulations to account for the interaction with the underlying substrate.

In Refs. [32, 30] Raman spectra were computed with the simplifying assumption of a model formed by a fragment of the full system, interacting with small Au clusters. That model provided a reliable interpretation of the experimental Raman spectra of both 1D and 2D systems, including the spectral changes observed upon interaction with the gold substrate, shedding light on the molecular phenomena involved. However, the choice of the fragment and the size and position of clusters must be carefully gauged, to avoid results dependent on the selected model. Here we propose and test a more general and less arbitrary scheme, accounting for the full lateral extension of the GDY, and keeping into account the substrate effects through the structural deformation induced on the GDY by the interaction with the metal substrate, although the last one is not explicitly included in the calculation of the Raman spectra. After successfully validating such an approach for the h-GDY test case, we turn our attention on the novel GDY systems on different metals, focusing on the study of the role of the GDY-substrate interaction in determining the vibrational properties and Raman spectra. 


\section{Methods}

We performed the on-surface synthesis of h-GDY in ultra high vacuum (base pressure of less than $5 \times 10^{-11}$ mbar. The $\mathrm{Au}(111)$ surface was prepared by repeated cycles of $\mathrm{Ar}^{+}$ion sputtering, followed by annealing at $720 \mathrm{~K}$. By means of an organic molecular evaporator (OME) at $304 \mathrm{~K}$, we evaporated the molecular precursor (1,3,5tris(bromoethynyl)benzene (tBEP)) on the cleaned $\mathrm{Au}(111)$ surface kept at room temperature (RT).

When deposited on $\mathrm{Au}$, terminal $\mathrm{Br}$ atoms in tBEP are released to form intermolecular connections through Au adatoms. At room temperature an organometallic system is formed, displaying the 2D network driven by the trigonal geometry of the precursor molecule. A subsequent thermal annealing at $480 \mathrm{~K}$ for about half an hour promotes the removal of $\mathrm{Au}$ adatom to form diacetylenic units by homocoupling reactions. Thermal treatment at higher temperatures leads to increased disorder in the network. We performed room-temperature STM measurements by means of an Omicron variable-temperature scanning tunneling microscope. STM images were taken in constant-current mode, with a chemically etched tungsten tip. We conducted ex-situ Raman measurements using a Renishaw InVia spectrometer coupled with an Argon laser $(514.5 \mathrm{~nm})$ with laser power of $5 \mathrm{~mW}$.

We carry out DFT calculations with the generalized gradient approximation (GGA) in the PBE form [35], and including van der Waals interactions between the organic overlayer and the substrate via a DFT-D2 Grimme potential [36]. We use the approach implemented in the SIESTA code [37] that relies on norm-conserving pseudopotentials and an atomic-orbitals basis set. This method allows us to treat fairly large unit cells, such as those formed by matching the considered GDY structures with the substrates, namely a $4 \times 4$ and a $5 \times 5$ supercell (see section 3.2.1). Moreover, it provides a firstprinciples characterization of the structural and electronic properties of the systems induced by the interaction with the substrate and allows us to generate simulated STM images using the Tersoff-Hamann approach [38].

We adopt a double-zeta basis set with polarization orbitals, a mesh cutoff for the real space grid of $400 \mathrm{Ry}$, and a $5 \times 5$ and $4 \times 4$ sampling of the Brillouin zone for the $4 \times 4$ and $5 \times 5$ surface reconstructions, respectively. A five times denser $\mathbf{k}$-space grid is used for the calculation of the density of states (DOS). We relax the organic layers and the first substrate layer until the forces reach the tolerance value of $0.04 \mathrm{eV}^{-1}$. The details of the calculation for h-GDY on $\mathrm{Au}(111)$ are reported in Ref. [30].

We compute the Raman spectra by DFT in a periodic boundary conditions (PBC) approach keeping a Gaussian basis set (GBS) (as implemented in the CRYSTAL package). Since DFT-PBC calculations of Raman intensities on a Gaussian basis sets cannot be adopted to describe metal surfaces, due to unavoidable convergence problems in the simulations, the computation of the Raman spectra have been carried out on the optimized crystal geometries of GDYs only, both in the free-standing case and for GDYs in the distorted configuration, as resulting from the interaction with $\mathrm{Au}$ and 

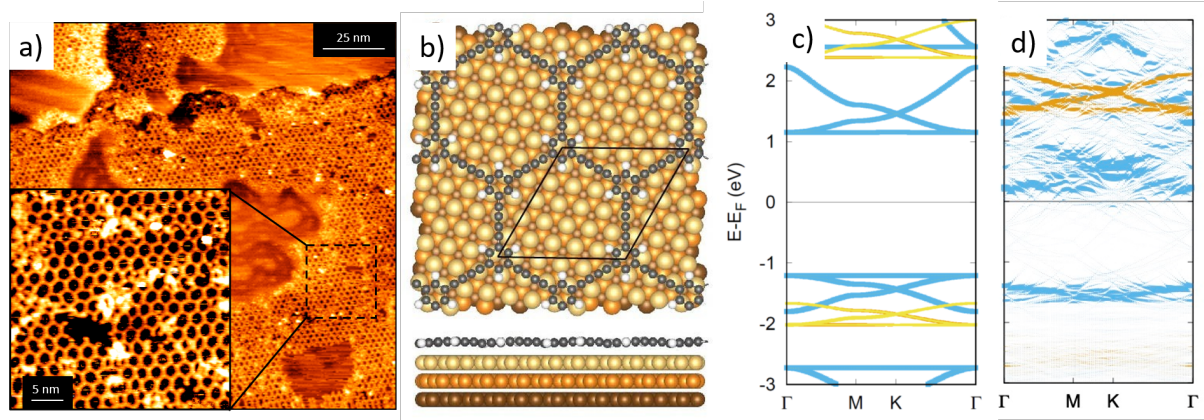

Figure 1. (a) STM images of h-GDY on $\mathrm{Au}(111)$ produced by on-surface synthesis. Inset: a magnification resolving the structure. Both images are collected at $-0.6 \mathrm{~V}$ and $0.3 \mathrm{nA}$. (b) Top and side view of our model for h-GDY on $\mathrm{Au}(111)$; the black line highlights the periodically repeated cell; this model was first adopted in [30]. (c) Band structure of the freestanding and (d) supported h-GDY. In panel (d) the states in blue and yellow/orange have $\mathrm{p}_{z}$ (orthogonal to the surface plane) and $\mathrm{p}_{x} / \mathrm{p}_{y}$ character, respectively.

Pt surface. These configurations are the optimized geometries as determined by the SIESTA calculations discussed above (keeping the same GGA functional also for the Raman calculations). By this procedure, it is thus possible to simulate the structural effects on the vibrational spectra of a $2 \mathrm{D}$ extended system as a consequence of the interaction with metal surfaces, avoiding more arbitrary and size-restricted molecular models formed by finite-size fragments interacting with small Au clusters.

As a benchmark for the adopted method, Fig. S4 of the Supporting Information reports the comparison of the Raman spectra obtained by DFT-PBC-GBS calculations of the free-standing systems here considered (namely, h-GDY, $\alpha$-GDY, and $\beta$-GDY) after full geometry optimization. The comparison involves the PBE functional adopted for most calculations here, and the PBE0 hybrid functional[39], which is considered the standard reference for Raman spectra of molecular systems [40, 21].

These two spectra are in agreement, presenting a very similar pattern in wavenumbers and intensities, confirming the reliability of the computational approach here adopted.

\section{Results and Discussion}

\subsection{Hydrogenated graphdiyne(h-GDY) on $A u(111)$ as an experimentally available system: theory and experiment}

In Ref. [30] a multi-level characterization of the properties of h-GDY on $\mathrm{Au}(111)$ based on structure investigation by STM and Raman spectroscopy was reported. We measured STM images on this system, reported in Figure 1a, showing the formation of an extended hexagonal structure on top of $\mathrm{Au}(111)$ surface. Figure $1 \mathrm{~b}$ reports the structural model, which is characterized by a $9^{\circ}$ rotation of the h-GDY network relative to the $[11 \overline{2}]$ surface direction. This same model could reproduce the experimental STM images [30]. The 


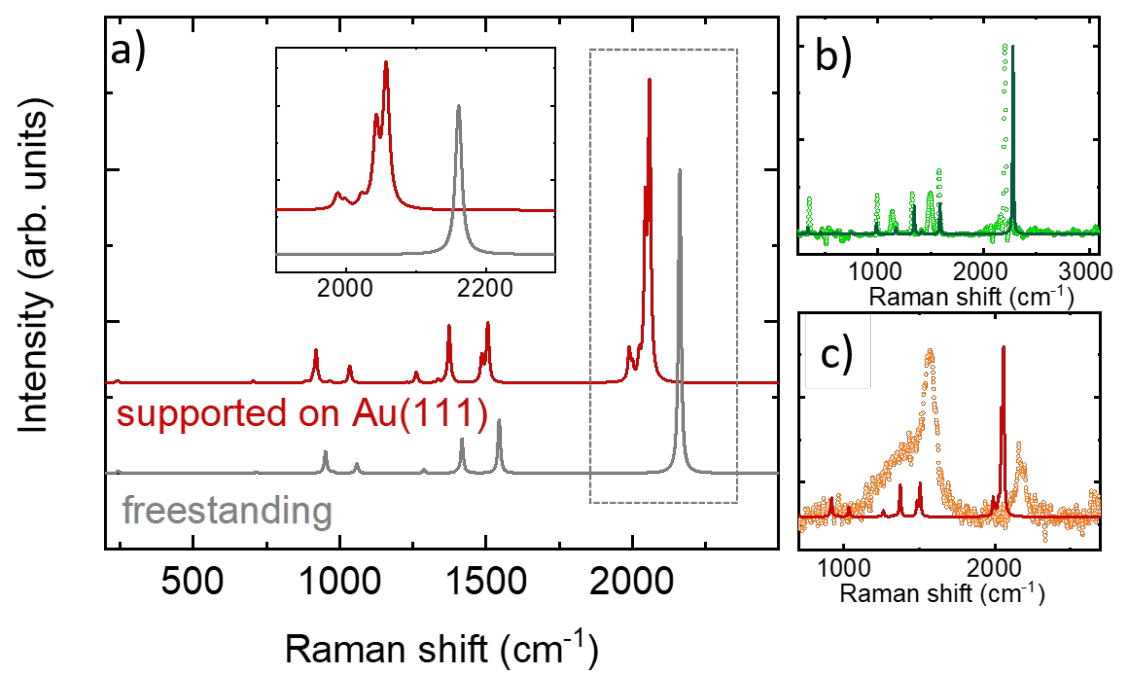

Figure 2. (a) DFT simulated Raman spectra of freestanding h-GDY and h-GDY on $\mathrm{Au}(111)$. Inset: a close-up of the spectral region of the ECC mode. (b) Comparison of DFT and experimental Raman spectra of the precursor molecule (tBEP). (c) Comparison of DFT and experimental Raman spectra of h-GDY system on $\mathrm{Au}(111)$.

structural relaxation induced by the interaction with gold results in a mild bending of h-GDY relative to the freestanding case.

Concerning the electronic properties, the band structure (Fig 11, $\mathrm{d}$ ) shows the downward energy shift of the empty p-states of the h-GDY, with a dramatic reduction of the electronic gap compared to the freestanding system (Fig,1:). Even though the energy gap between $\mathrm{p}_{z}$ bands remains visible, the charge transfer from the $\mathrm{Au}(111)$ surface to the h-GDY turns the carbon network into a weakly metallic system, with originally empty $\mathrm{p}_{z}$ states downshifted in energy and touching the Fermi level.

Figure 22 reports the Raman spectra for the h-GDY system, carried out by using the approach presented above, for both the freestanding system and for h-GDY supported on $\mathrm{Au}(111)$. DFT simulations tend to systematically overestimate the conjugation giving different peak frequencies compared to experiment, by a roughly constant factor. It is a standard and widely adopted method to rescale the simulated frequencies using a scaling factor evaluated by comparison with experimental vibrational frequencies. However, Figure $2 \mathrm{~b}$ shows a very good agreement in the comparison of the experimental and simulated spectra, in particular the almost coinciding frequency of the stretching mode of the phenyl ring at $1581 \mathrm{~cm}^{-1}$, indicating no need of any rescaling. Hence all the simulated Raman spectra reported in this work are unscaled.

When h-GDY interacts with the Au surface, peculiar effects are found in the Raman spectra. In particular, the peak above $2000 \mathrm{~cm}^{-1}$, assigned to the collective CC stretching of the sp-domains (the so-called Effective Conjugation Coordinate ECC mode of polyynes [21, 8]), exhibits an evident downshift upon interaction with $\mathrm{Au}$ compared to the freestanding case. In addition to a frequency shift, new features 
appear in the region of the ECC band when the system is supported on $\mathrm{Au}(111)$, relative to the single peak observed for the freestanding system. This single peak is in fact the convolution of three contributions associated to different Raman-active combination of the ECC modes on the different diacetylenic units in the cell, almost coincident in wavenumber. Conversely the h-GDY interacting on $\mathrm{Au}$ exhibits distinctive features between 1980 and $2060 \mathrm{~cm}^{-1}$ of which three are again the most intense ones $(2022,2043$, and $2057 \mathrm{~cm}^{-1}$, see inset of Fig 2a). Inspection of the normal modes reveals that these most intense bands are still related to collective ECC vibrations on the diacetylenic units, while the weaker bands at lower wavenumbers (1988 and $2000 \mathrm{~cm}^{-1}$ ) are CC stretching vibrations more localized on specific bonds in the sp-carbon domains. This is in agreement with previous works in which also the pattern or normal modes was found to be affected by the specific interaction with gold. In particular, the collective ECC mode on the diacetylenic units changes into more localized CC stretching vibrations. At frequencies below $1600 \mathrm{~cm}^{-1}$, vibrations of the aromatic units are found, but they are not so significantly influenced by the interaction with the gold surface.

In previous works [32, 30] the observed frequency downshift of the ECC mode has been interpreted as the result of the strong interaction with $\mathrm{Au}$ which is responsible for weakening the $\mathrm{CC}$ bonds, thus lowering their stretching force constant and causing lower vibrational wavenumbers. In the case of finite-length sp-carbon wires (i.e., polyynes), a downshift of the ECC mode is usually correlated to a more equalized structure (i.e., a smaller bond-length alternation - BLA - between the quasi-single and quasi-triple CC bonds) and also a smaller band gap, or as a consequence of tensile strain [41]. By computing the BLA of the di-acetylenic units in the h-GDY network (as the difference between the average bond lengths of the single $\mathrm{CC}$ bonds and the bond length of the triple CC bond at the center of the $s p$-carbon chain), values of $0.123 \AA$ and $0.119 \AA$ are found for the freestanding and $\mathrm{Au}$-supported systems, respectively. The difference between these two values $(0.004 \AA)$ is however not sufficient to justify the downshift observed in the Raman spectra. Analyzing the value of the length of the different CC bond in the sp-carbon units, in the Au-supported system longer values are found, with all bonds longer by 0.01-0.02 $\AA$ than in the free-standing case. The interaction with gold hence reduces the bond strength of the $\mathrm{CC}$ bonds in the sp-carbon units, thus implying softer stretching force constants and justifying the downshift in wavenumber of the related stretching vibrational modes. These results are consistent with those discussed in [32, 30], using DFT on molecular fragments interacting with small Au clusters. The spectral changes found in previous investigations also indicated a change of normal modes upon interaction with gold, namely the formation of more localized CC stretching vibrations induced by the presence of gold. This led to the appearance of peaks well below $2000 \mathrm{~cm}^{-1}$ (1939 and $1969 \mathrm{~cm}^{-1}$ ), which are hardly observed in the experimental spectrum [30]. The main features of the experimental Raman spectrum are well reproduced by the current computational approach (see Fig.2.). The splitting of the ECC mode into many different peaks all in a small spectral range can account for the broad band observed in the experiment. However, a mismatch is present when 
considering the experimental and simulated spectrum of h-GDY on $\mathrm{Au}(111)$. The slightly different peak frequency of ECC mode in simulated and experimental spectra can be related to the well-known difficulties of DFT in precisely estimating the degree of conjugation. In addition, the experimental spectrum shows a broad and intense band in the $\mathrm{sp}^{2}$-carbon region below $1600 \mathrm{~cm}^{-1}$, which is reasonable considering the presence of defects and disorder, not included in the simulation and giving rise to the so-called $\mathrm{G}$ and $\mathrm{D}$ features typical of $\mathrm{sp}^{2}$ amorphous carbon.

\section{2. $\alpha$ - and $\beta$-graphdiynes on metals}

3.2.1. Structural models and electronic properties. The PBE-relaxed freestanding structures of $\alpha$-GDY and $\beta$-GDY exhibit periodicities of $11.493 \AA$ and $14.704 \AA$, respectively (slightly larger than obtained in PBE0, namely $11.398 \AA$ and $14.600 \AA$, respectively). They can be matched with the same supercell for both substrates, given their similar lattice constants: $4.21 \AA$ for $\mathrm{Au}$ and $4.18 \AA$ for Pt in our PBE model. In particular, we adopt a structural model in which $\alpha$-GDY is matched to a $4 \times 4$ supercell of the substrate and $\beta$-GDY to a $5 \times 5$ one. The resulting strain of the GDY structures is $3.6 \%(2.9 \%)$ and $1.2 \%(0.5 \%)$ for $\alpha$-GDY ( $\beta$-GDY) on $\mathrm{Au}(111)$ and $\mathrm{Pt}(111)$, respectively. The sp-carbon linking chains of the $\alpha$-GDY are aligned along the $[11 \overline{2}]$ directions of the two surfaces (see Figure $3 a, b$ ), while in the $\beta$-GDY they follow the surface [1 $1 \overline{1} 0]$ directions and appear rotated by $30^{\circ}$ relative to the former (see Fig. 3r,d).

Both $\alpha$-GDY and $\beta$-GDY show a fairly small structural relaxation on $\mathrm{Au}(111)$, contrasted with the remarkable distortion on $\mathrm{Pt}(111)$, due to a stronger interaction with the Pt substrate. In particular, $\alpha$-GDY remains almost flat on $\mathrm{Au}(111)$ (the spread in the $z$ coordinate, orthogonal to the surface, is $0.18 \AA$ ), with an average distance of $2.64 \AA$ from the outermost surface layer, while on Pt(111) the GDY is more corrugated $(0.63 \AA)$, and lies at $\mathrm{a} \simeq 23 \%$ smaller average distance from the surface $(2.02 \AA)$ 国

This difference between the two substrates is confirmed also in the $\beta$-GDY case. The carbon network, although slightly more corrugated than the $\alpha$-GDY system, is still almost flat on $\mathrm{Au}(111)$, with a corrugation of $0.31 \AA$. When deposited on $\operatorname{Pt}(111)$ the $\beta$-GDY structure becomes instead largely distorted, both in-plane and out-of-plane: we observe a deformation of the $s p$-carbon units and an overall vertical corrugation of $0.98 \AA$. By comparing $\alpha$-GDY with $\beta$-GDY one can note that the latter sits slightly farther from the surface (average distance $2.73 \AA$ on $\mathrm{Au}(111)$ and $2.21 \AA$ on $\mathrm{Pt}(111)$ ), suggesting a weaker interaction with the substrate.

The larger distortion of the $\beta$-GDY compared to the $\alpha$-GDY can be understood in terms of the different orientation of the $s p$-chains relative to the underlying substrates. Indeed in $\beta$-GDY the interaction with atoms of the top surface layer is favored by the alignment along the $[1 \overline{1} 0]$ crystalline lines.

$\ddagger$ The average distance is obtained as the difference between the mean $z$ coordinate of the GDY and of the uppermost surface layer. The corrugation is determined as the difference between the maximum and the minimum value of the $z$ coordinates in the overlayer. 


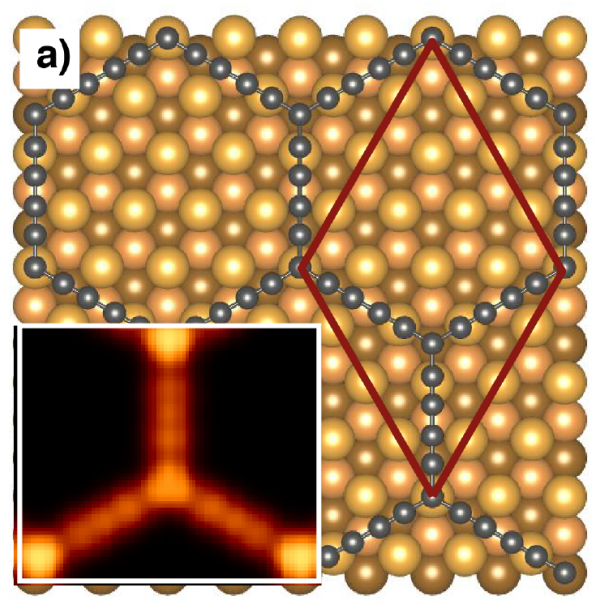

$2.64 \AA$

000000000000000000000

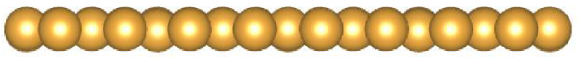

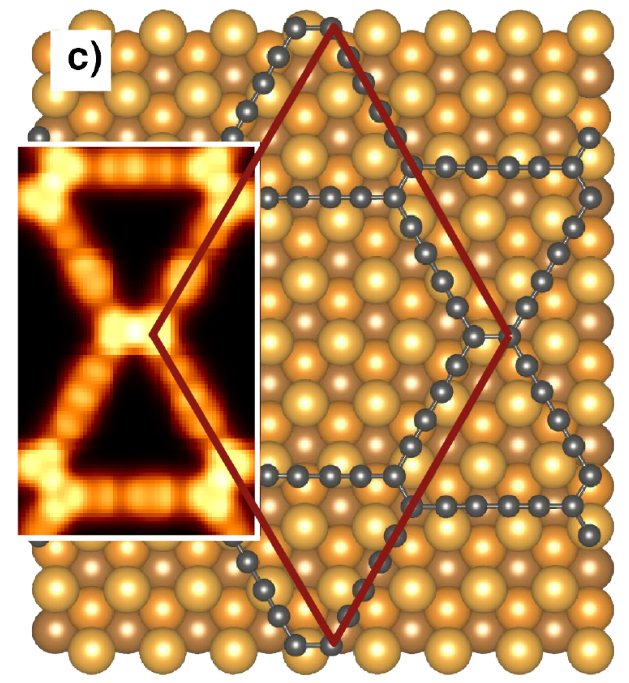

$2.73 \AA$

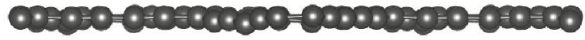

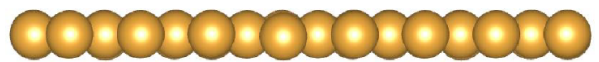

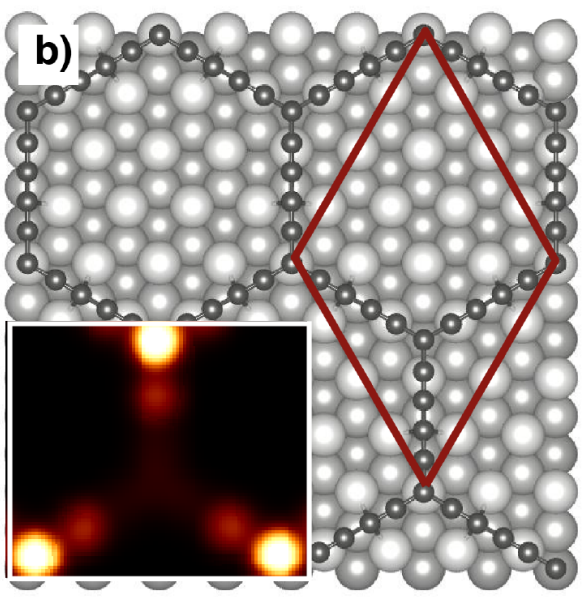

$00000 \cdot 0000000000000092.02 \AA$ 00200000000000
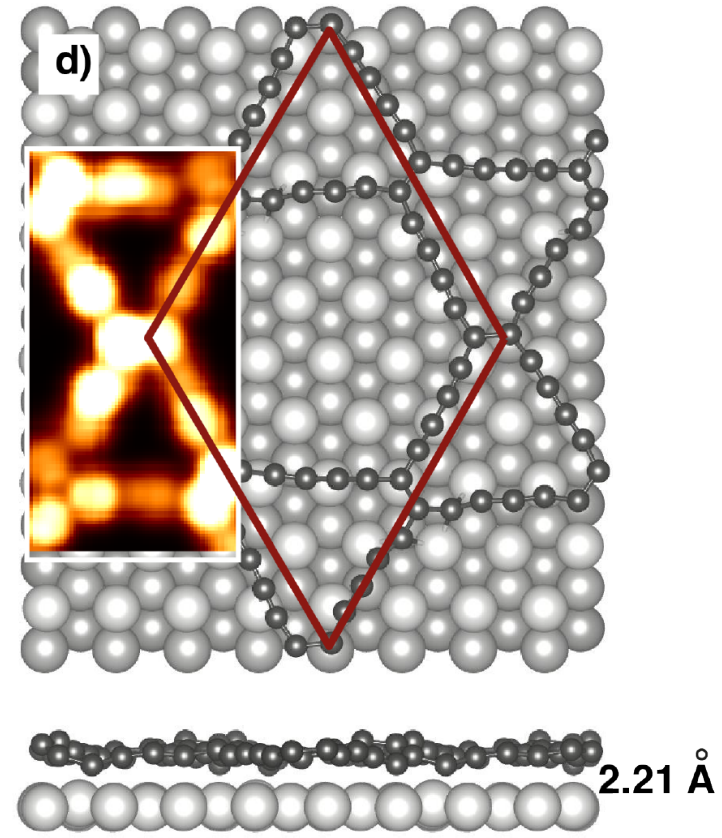

Figure 3. Top view and side view (two outermost layers only) of (a) $\alpha$-GDY on $\mathrm{Au}(111)$, (b) $\alpha$-GDY on Pt(111), (c) $\beta$-GDY on Au(111), and (d) $\beta$-GDY on Pt(111). The average distance between the GDY and the surface layer is indicated, and the unit cell is marked in red. Atoms in the first-, second- and third- substrate layer are marked with different shades. The simulated STM images obtained for filled states (integration range: from $0.5 \mathrm{eV}$ below the Fermi energy $\epsilon_{\mathrm{F}}$ up to $\epsilon_{\mathrm{F}}$; tip distance: $2 \AA$ from the GDY average plane) are reported as aligned insets. The color scale of STM images is the same for all the panels, ranging from 0.0 to $0.0035 \mathrm{e} / \mathrm{Bohr}^{3}$. 
The insets of Fig. 3 report the simulated STM images of the occupied states (bias $V=-0.5 \mathrm{~V}$ ). These four images, taken at the same height above the average $z$ of the organic layer and reported in the same color scale, exhibit a different contrast on the GDY layer, depending on the substrate on which they are adsorbed. In $\alpha$-GDY/Au the whole hexagonal structure of the GDY is visible, including contributions also from the sp-carbon chains, while in $\alpha$-GDY/Pt the extra-bright contribution of the $\mathrm{C}$ atoms at the corners of the GDY porous structure emerges prominently. Notably, the brightest atoms sit on top of the substrate FCC sites and are less protruding toward vacuum.

The STM contrast is indeed mainly due to an electronic effect, being these atoms characterized by an excess of electrons. The STM simulation of $\beta$-GDY on $\mathrm{Au}(111)$, obtained with identical setting as adopted for $\alpha$-GDY, is overall brighter, due to the larger charge transfer toward the organic layer (see below), and displays bright features in correspondence to the carbon atoms at the corners of the starry structure, and a relatively intense contrast also along the sp-carbon chains. Also in this case the dominant effect is electronic: the height modulation is indeed relatively small, while the electronic charge accumulated on CC single bonds in the chain and at the corners of the structure is three times larger than the excess charge on triple $\mathrm{C} \equiv \mathrm{C}$ bonds. The STM image for $\beta$-GDY on $\mathrm{Pt}(111)$ is characterized by blurred spots in correspondence of the $\mathrm{C}$ atoms that interact most strongly with the underlying substrate, leading to bright features arising from a combination of structural deformation (atoms displaced toward vacuum) and electronic effect (charge transfer). The more intense STM signal of GDY on Pt than on $\mathrm{Au}$, reaching saturation on certain atoms in the adopted scale, reflects a larger local transfer of electrons on platinum (see details in section 3.2.2), giving a further evidence of the larger strength of the interaction with this substrate.

The total-energy analysis confirms that $\alpha$-GDY is more strongly bound to $\mathrm{Pt}(111)$ than to $\mathrm{Au}(111)$. Indeed, the adsorption energy per carbon atom is $-0.38 \mathrm{eV}$ on $\mathrm{Au}$ and $-0.48 \mathrm{eV}$ on Pt (see Table S1 in the Supporting Information for more details and definition of the relevant quantities). It is worth noting that the energy required to strain and distort the GDY on $\mathrm{Au}(0.078 \mathrm{eV} /$ atom $)$ is about half that on $\mathrm{Pt}(0.14 \mathrm{eV} / \mathrm{atom})$, due to the larger distortion on $\mathrm{Pt}$, and despite the smaller strain required to match the GDY to the Pt substrate spacing. On the other hand, the pure-adhesion energy contribution is $-0.46 \mathrm{eV} /$ atom on $\mathrm{Au}$ and $-0.62 \mathrm{eV}$ on Pt.

The adsorption energy of $\beta$-GDY on both substrates follows similar trends as for $\alpha$-GDY. The energy cost of strain+distortion is slightly smaller $(0.02 \mathrm{eV} /$ atom on $\mathrm{Au}$ and $0.12 \mathrm{eV} /$ atom on $\mathrm{Pt}$ ). The total adsorption energies are the same as for $\alpha$-GDY $(-0.38 \mathrm{eV}$ on $\mathrm{Au}$ and $-0.48 \mathrm{eV}$ on $\mathrm{Pt})$, with pure-adhesion contributions $(-0.40 \mathrm{eV} /$ atom on $\mathrm{Au}$ and $-0.60 \mathrm{eV} /$ atom on $\mathrm{Pt})$ that are also slightly smaller.

3.2.2. Electronic properties The stronger interaction of GDYs with Pt compared to Au manifests itself also in the changes induced on the electronic properties in the adsorbed system. The band structure of the $\alpha$-GDY on $\mathrm{Au}(111)$, reported in Figure $4 \mathrm{~b}$, shows a shift of the Dirac cone relative to the freestanding case (Fig, 4 a). This -0.5 eV energy 

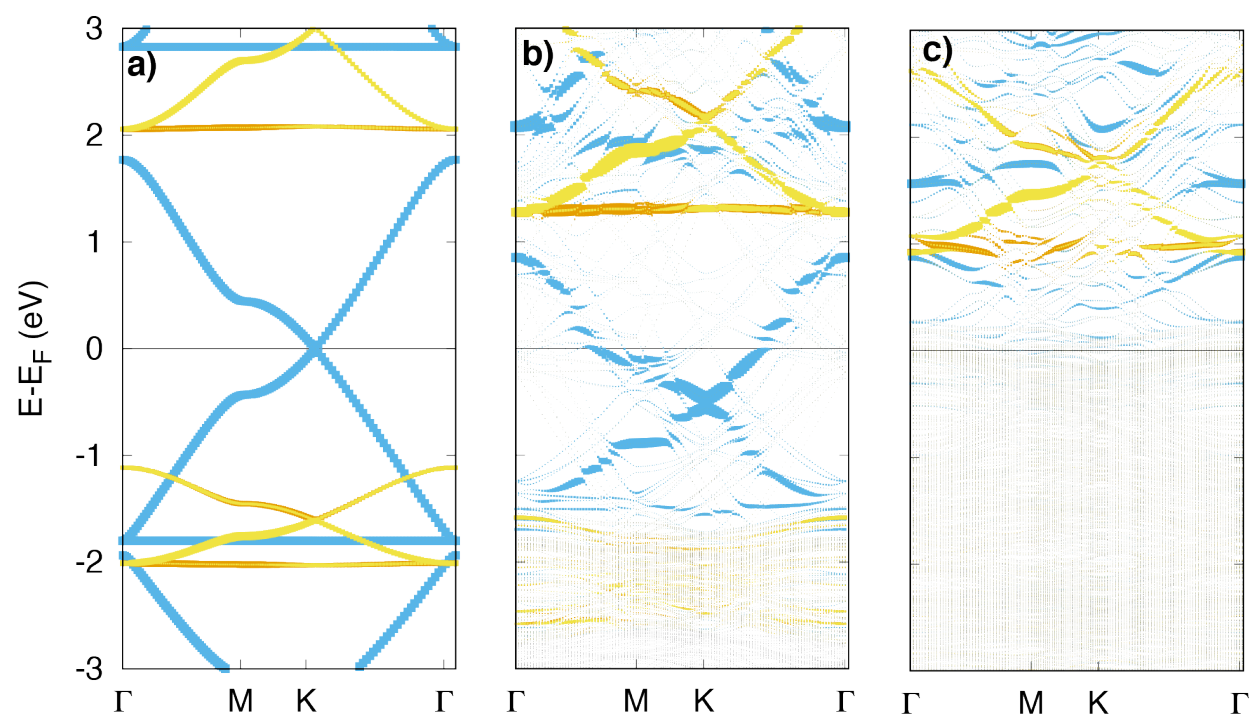

Figure 4. Band structure of $\alpha$-GDY in the following configurations: (a) freestanding; (b) adsorbed on $\mathrm{Au}(111)$; (c) adsorbed on $\mathrm{Pt}(111)$. The $\mathrm{p}_{z}$ weights are highlighted in blue and the $\mathrm{p}_{x} / \mathrm{p}_{y}$ ones in yellow.

shift is accompanied by the opening of a small $0.1 \mathrm{eV}$ gap of (see also Figure $\mathrm{S} 2$ in the SI) In contrast, the interaction with Pt(111) washes the Dirac-cone feature away: it mostly disappears in the dense spectrum of the filled states of the underlying Pt surface. In fact, by zooming around the Fermi level, one can still detect a reminiscence of the Dirac cone just above the Fermi level, with upward and downward dispersing bands with relevant $\mathrm{p}_{z}$ character (see Figure $\mathrm{S} 2$ in $\mathrm{SI}$ ).

The dispersion of the $\mathrm{p}_{x}, \mathrm{p}_{y}$ bands is moderately modified by the interaction with $\mathrm{Au}(111)$ : the main visible effects is indeed a downward energy shift mostly of the empty bands. Differently, on Pt(111) the distorted GDY structure causes a substantial mixing with $\mathrm{p}_{z}$ states, leading for example to a more dispersive behavior of the band near $+1 \mathrm{eV}$, which is otherwise flat in the freestanding GDY.

The opposite energy shift of the filled bands of $\alpha$-GDY on the two substrates is an effect of the opposite charge transfers occurring in the adsorbed GDYs. The analysis of the Mulliken charges evidences a transfer of 0.018 electrons/C atom from $\mathrm{Au}(111)$ to $\alpha$-GDY and, conversely, of 0.01 electrons/C atom from the $\alpha$-GDY to the Pt substrate.

Notably, despite such an average electron transfer from $\alpha$-GDY to $\operatorname{Pt}(111)$, certain $\mathrm{C}$ atoms in the structure receive contributions with opposite sign. In particular the $\mathrm{C}$ atoms sitting in hollow positions are characterized by an excess of electron charge ( $\sim 0.08$ electrons $)$ compared to the freestanding system, resulting in bright spots in STM images. Differently, the sp $\mathrm{C}$ atoms forming the chains are characterized by the largest reduction of charge $(\sim-0.07$ electrons). On both substrates, due to the interaction with the metal and the ensuing charge transfer, $\alpha$-GDY acquires a frank metallic character, as evidenced also by the density of states (DOS) reported in SI, Figure S3.

The freestanding $\beta$-GDY is a semiconductor with a gap of $0.26 \mathrm{eV}$, see Fig. 5 a. 

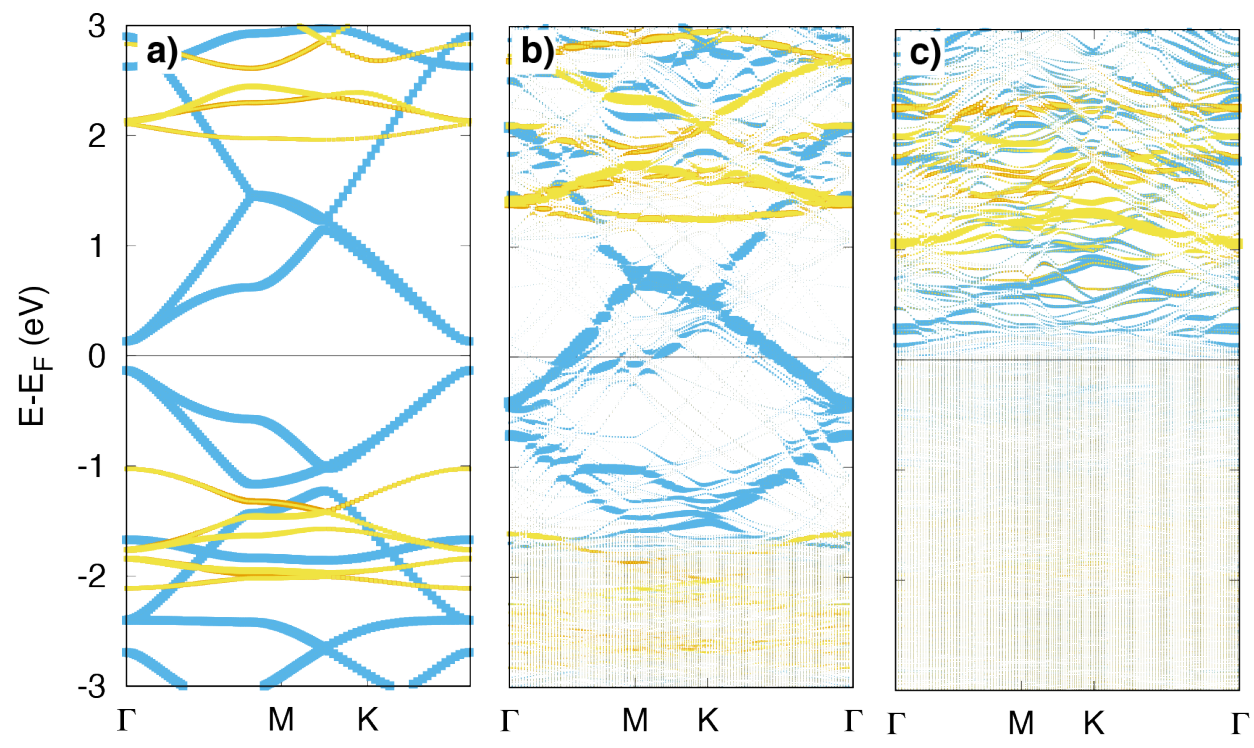

Figure 5. Same as Fig. 4 , but for $\beta$-GDY.

When adsorbed on $\mathrm{Au}(111) \beta$-GDY maintains the main features of its band structure, with the bands downshifted in energy by $\sim 0.5 \mathrm{eV}$, as reported in Fig. 5b. Due to this shift, the $\beta$-GDY $\mathrm{p}_{z}$ states cross the Fermi level leading to a metallic character in the overlayer, as confirmed also by the DOS (see Figure S3 in SI).

The effect of such charge transfer includes a displacement of the Dirac-cone-like feature that can be seen at $+1.3 \mathrm{eV}$ in the freestanding GDY, moving it closer to the Fermi level.

If a similar charge transfer was observed on a semiconducting/insulating substrate, it could be controlled via an electric field and exploited for switched electronic transport in a gated device setup. Like for $\alpha$-GDY, the $\mathrm{p}_{x}$ and $\mathrm{p}_{y}$ bands are relatively weakly modified upon adsorption on $\mathrm{Au}$, apart for the downward energy shift discussed above. On Pt(111), instead, the strong interaction and hybridization with the substrate states completely modifies the bands of the $\beta$-GDY overlayer (see also the broadening of the DOS in the SI, Figure S3).

The effect of the structural deformation on the $\beta$-GDY bands is less important than that of the hybridization with the metallic bands. This is confirmed also by the bandstructure calculation of a freestanding $\beta$-GDY frozen in the distorted configuration induced by the $\operatorname{Pt}(111)$ substrate, which shows bands rather similar to those of the fully optimized (flat) freestanding structure (see SI, Figure S1). Also in this case the changes of the states at the Fermi level due to the interaction with the substrate confer a metallic character to the carbon overlayer. The downshift of the conduction band of both GDYs below the Fermi level on the gold substrate and the strong hybridization with the platinum substrate states suggest that there is no vertical barrier for the flow 
of electrons at the interface, in analogy with what is found for $\gamma$-GDY on different metal surfaces [29].

The Mulliken charge analysis evidences a charge transfer of 0.013 electrons/C atom from $\mathrm{Au}$ surface to the $\beta$-GDY, in agreement with the observed shift of the bands, while on $\mathrm{Pt}(111)$ a negligibly small average charge (0.0006 electrons/C atom) is transferred from the overlayer to the surface. Like in the $\alpha$-GDY case, the individual $\mathrm{C}$ atoms in the structure give contributions with opposite sign. The maximum charge transfer on a single atom amounts to 0.06 electrons/C atom.

3.2.3. Vibrational properties and simulated Raman spectra. Based on the validation obtained for h-GDY, we have applied the same computational approach to $\alpha$ - and $\beta$-GDY polymorphs and their interaction with both $\mathrm{Au}$ and $\mathrm{Pt}$ metal surfaces. The DFT-computed Raman spectra for these systems are reported in Figure 3 , including a comparison with the freestanding spectra.

Considering first the freestanding $\alpha$-GDY, three main bands are predicted by the simulations. The most intense one, located at $1950 \mathrm{~cm}^{-1}$, is indeed the convolution of two intense bands, both of which are related to normal modes consisting in different Raman-active combinations of the ECC modes on the single diacetylenic sp-carbon units. Another intense band is found at about $1420 \mathrm{~cm}^{-1}$ and it is the convolution of two different bands (at 1420 and $1423 \mathrm{~cm}^{-1}$ respectively), related to stretching of the $\mathrm{C}-\mathrm{C}$ bonds between the $\mathrm{sp}^{2}$ carbon atoms and the first sp carbon atoms of the diacetylenic units. A weaker band is predicted as a convolution of the two bands at 784 and $785 \mathrm{~cm}^{-1}$, and it is due mainly to CCC bending modes involving $\mathrm{sp}^{2}$ carbon atoms. A further, and even weaker band is found at about $550 \mathrm{~cm}^{-1}$, associated to linear bending modes of the sp-carbon domains.

Our calculations predict substantial modifications when $\alpha$-GDY interacts with $\mathrm{Au}$ and Pt surfaces. The two strongest bands undergo a large frequency shift. The ECC bands at $1949 \mathrm{~cm}^{-1}$ downshifts to $1652 \mathrm{~cm}^{-1}$ upon interaction with Au and to $1523 \mathrm{~cm}^{-1}$ upon interaction with Pt. Similarly, the $1421 \mathrm{~cm}^{-1}$ band (i.e., CC stretching between $s p^{2}$ and $s p \mathrm{C}$ atoms) moves down to $1154 \mathrm{~cm}^{-1}$ on $\mathrm{Au}$ and to $1178 \mathrm{~cm}^{-1}$ on $\mathrm{Pt}$. Furthermore, the bands associated to CCC bending modes shift from $784 \mathrm{~cm}^{-1}$ to $730 \mathrm{~cm}^{-1}$ on $\mathrm{Au}$ and $731 \mathrm{~cm}^{-1}$ on Pt. The interaction with the surface seems to affect more strongly the sp-carbon domains in the case of Pt, inducing a larger downshift of the ECC bands with respect to Au. On the other hand, the geometry of GDY around the $\mathrm{sp}^{2}$ carbon atoms is less affected on $\mathrm{Pt}$, as demonstrated by the smaller downshift found for the $1421 \mathrm{~cm}^{-1}$ band compared to the Au case. In addition to the bands already present in the Raman spectrum of the free-standing $\alpha$-GDY, when supported a further peak arises at $1802 \mathrm{~cm}^{-1}$ (weak) on $\mathrm{Au}(111)$ and at $1742 \mathrm{~cm}^{-1}$ on $\operatorname{Pt}(111)$, with an appreciable intensity. These bands are associated to CC stretching vibrations involving the $\mathrm{CC}$ triple-bonds: these lower-frequency normal modes are the counterpart of the so-called " $\beta$ " mode, observed and predicted for finite-length polyynes [ $[$, 21]. The larger intensities found on $\mathrm{Pt}$ indicate again that in this case the interaction with the 


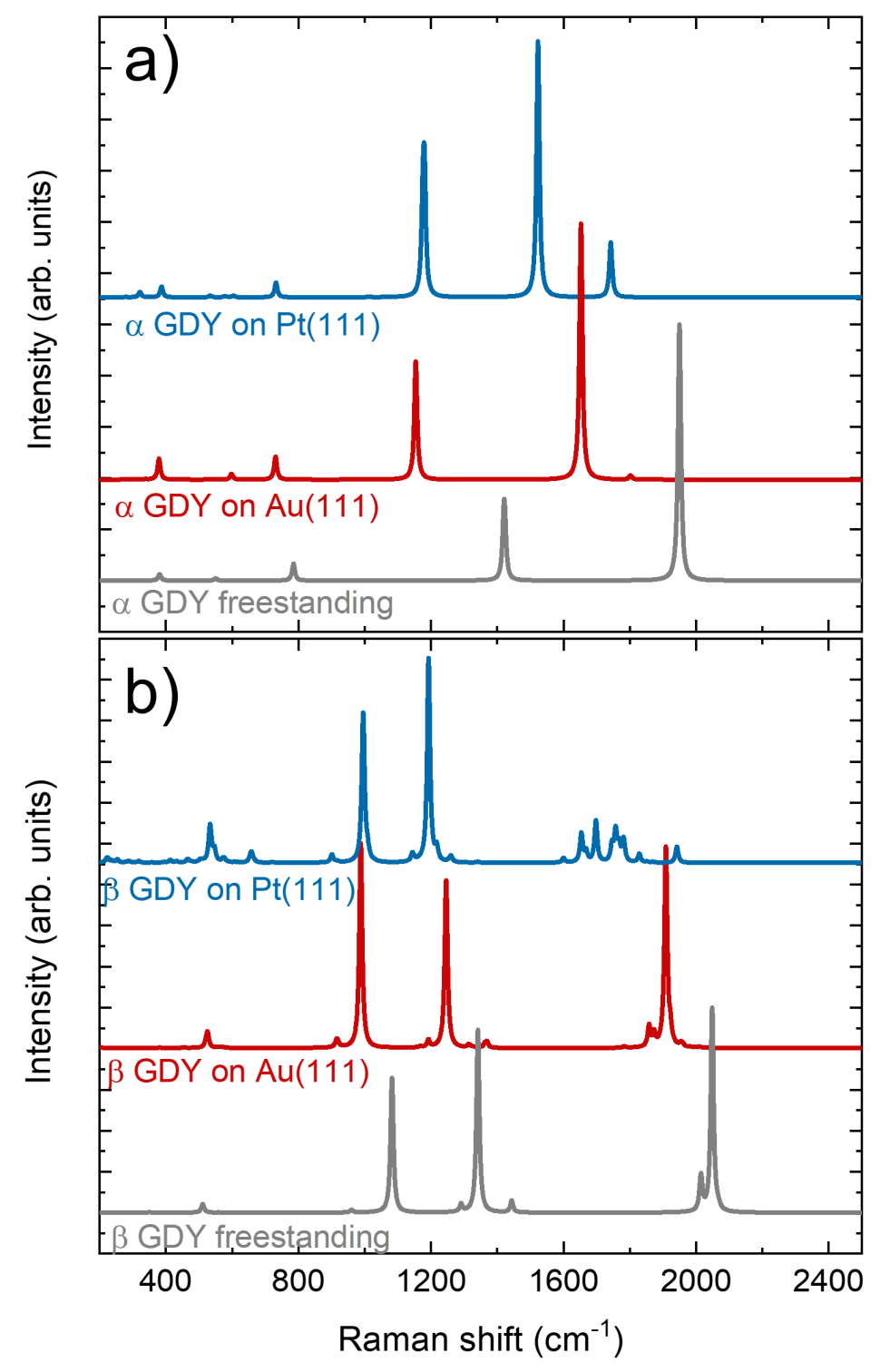

Figure 6. (a) DFT-simulated Raman spectra of $\alpha$-GDY freestanding and supported on $\mathrm{Au}(111)$ and on $\mathrm{Pt}(111)$. (b) DFT simulated Raman spectra of $\beta$-GDY freestanding and supported on $\mathrm{Au}(111)$ and on $\mathrm{Pt}(111)$.

metal more strongly affect the $s p$-carbon domains. In order to rationalize this spectral modulation, bond-lengths and BLA values have been analyzed. BLA values on the $s p$ carbon domains change from $0.095 \AA$ in the free-standing, to $0.11 \AA$ on Au and to $0.078 \AA$ on Pt. The lack of a clear trend in these values reveals that the downshifts predicted on metal surfaces cannot be simply related to the degree of conjugation usually leading to more equalized diacetylenic units [42]. On the other hand, the CC bond-lengths on the diacetylenic units become significantly longer upon interaction with the metal surfaces, with elongations by up to $0.066 \AA$. This allows us to conclude that, also in this case, the 
interaction with $\mathrm{Au}$ and $\mathrm{Pt}$ surfaces gives more antibonding character to the $\mathrm{CC}$ bonds, weakening the bond strength, and thus resulting in lower $\mathrm{C}-\mathrm{C}$ stretching wavenumbers.

Considering now the $\beta$-GDY polymorph, the freestanding configuration exhibits four main bands plus a few weaker ones. The most intense band at $2048 \mathrm{~cm}^{-1}$ is associated to ECC vibrations on the diacetylenic units. Two other weaker contributions forming the band at $2015 \mathrm{~cm}^{-1}$ are also associated to Raman active combinations of ECC vibrations on different diacetylenic units. The other intense band at $1341 \mathrm{~cm}^{-1}$ is mainly related to the stretching mode of the $\mathrm{CC}$ bond between adjacent $\mathrm{sp}^{2}$ carbon atoms. $\mathrm{A}$ fourth intense band is computed at $1082 \mathrm{~cm}^{-1}$ due to CCC bending modes involving $\mathrm{sp}^{2}$ carbon atoms. As a consequence of the interaction with the Au surface, similar modifications as for $\alpha$-GDY are observed. All the intense bands display a consistent downshift in frequency, while keeping the same assignment in terms of normal modes of vibration. Such downshift is ascribed to a weakening of the $\mathrm{CC}$ bond of the network and a softening of the vibrational stretching force constants, as discussed for the $\alpha$ GDY case. In particular, at the corners of the structure bond lengths increase from $1.41 \AA$ in the freestanding to $1.43 \AA$ and $1.44 \AA$ on $\mathrm{Au}$ and $\mathrm{Pt}$, respectively. Triple and single bonds in the sp-carbon units elongate when moving from freestanding $(1.25 \AA$ and $1.34 \AA$, respectively) to $\mathrm{Au}(1.26 \AA$ and $1.36 \AA)$ and $\mathrm{Pt}(1.30 \AA$ and $1.35 \AA)$ supported structure. However, the resulting BLA value increases when the overlayer is on $\mathrm{Au}$ (from $0.097 \AA$ to $0.099 \AA$ ) while decreases on Pt (from $0.097 \AA$ to $0.050 \AA$ ). A complex interplay between $\pi$-electron conjugation and structural parameters occurs also in this case, showing that changes in conjugation can have a non trivial effect on the BLA [43].

Conversely, when interacting with Pt, the Raman spectrum shows a more complicated pattern, in particular in the region between 1500 and $2000 \mathrm{~cm}^{-1}$ where many bands of comparable intensities are predicted, spanning an extended range of wavenumbers. This is due to the fact that, as shown in Figure 3 , the structure of $\beta$-GDY is strongly deformed upon interaction with the $\mathrm{Pt}$ surface, and some of the diacetylenic units deviate significantly from linearity, showing bending angles of about $160^{\circ}$. As a consequence of this structural deformation, the normal modes in this region are not anymore acting as a collective CC stretching mode delocalized across the whole sp-carbon units as in the freestanding crystal, but are now localized on different CC stretching vibrations along the distorted sp-carbon units, each mode usually involving mainly one or a few $\mathrm{CC}$ bonds. Hence a multiplicity of bands arises due to the variety of different $\mathrm{CC}$ bond stretching, resulting from the large structural deformation induced by the Pt surface.

Based on these results, Raman spectroscopy confirms its usefulness for the characterization of GDYs and other hybrid $\mathrm{sp}-\mathrm{sp}^{2}$ carbon materials, providing important information on their structural properties and on the effects of the interaction with metal surfaces. The strong sensitivity of the Raman features on small changes of the molecular structure allows one to use this technique to investigate subtle effects, related to both the inherent structure of the carbon 2D network and to its interactions with the substrate. 


\section{Conclusions}

Building on recent experimental and theoretical results on h-GDY [30], we unveil the mechanisms underlying the changes of electronic and vibrational properties of different GDYs induced by the presence of a metal substrate. The present work aims at predicting properties that will be easily measured as soon as the so-far-scarcely-characterized $\alpha$-GDY and $\beta$-GDY on Au and Pt are synthesized and studied experimentally. In particular, we show that the Dirac cones that characterize the semimetallic free-standing $\alpha$-GDY undergo an energy shift in the interaction with an Au substrate, while they completely disappear in the case of Pt. The $\beta$-GDY polymorph, instead, which is semiconducting in its free-standing form, becomes metallic when deposited on both $\mathrm{Au}$ and Pt surfaces. Overall, this analysis confirms the possibility to tune the electronic properties of the GDYs by appropriately choosing the substrate. In particular, the choice of $\mathrm{Au}(111)$ allows one to preserve the main features of the GDY band structure while substrates with strongly-interacting d-states at the Fermi level, such as $\operatorname{Pt}(111)$, induce strong deformation of both the structural and electronic properties of the $2 \mathrm{D}$ carbon network.

Such important changes in the electronic structure are accompanied by dramatic effects on the predicted Raman spectra. Since full Raman simulations for extended GDYs on metal substrates are known to suffer from severe limitations, in the present work we proposed a simplified approach: we keep the geometry of the adsorbed GDY, and remove the metal surface altogether: this approach retains those effects on the Raman spectra which can be ascribed to the structural changes that the GDYs undergo in the presence of the metallic substrate, while leaving out charge-transfer and substrateGDY covalency effects.

We successfully validate this approach on the $\mathrm{Au}(111)$-synthesized h-GDY case, whose experimental Raman spectra are available. By applying this method to $\alpha$ - and $\beta$ GDY, we observe Raman features which are consistent with the structural modifications induced by the presence of the metal substrate such as frequency shifts and splittings of the ECC normal modes, thus confirming that Raman spectroscopy can provide a wealth of information for GDYs on metals.

The importance of sp-sp ${ }^{2}$ bidimensional overlayers, such as GDYs, has been recently highlighted in view of their potential technological applications, which could exploit their peculiar functional properties [44]. In this framework, predictions of the substrate effect are crucial, since substrates themselves provide additional degrees of freedom to the engineering of novel 2D materials. A clear understanding and control of the GDYsubstrate interactions, will provide us with a suitable spectrum of choices of both the GDY polymorph and the underlying substrate, thus opening the way toward a controlled tuning and engineering of the properties of the organic overlayer. 


\section{Acknowledgments}

A.M., F.T., and C.S.C. acknowledge funding from the European Research Council (ERC) under the European Union's Horizon 2020 research and innovation program ERC Consolidator Grant (ERC CoG2016 EspLORE grant agreement no. 724610, website: www.esplore.polimi.it). S.A., G.F., N.M., and G.O. acknowledge CINECA for the use supercomputing facilities under the agreement with UNITECH-INDACO. N.M. acknowledges support from the grant PRIN2017 UTFROM of the Italian Ministry of University and Research.

\section{References}

[1] Hirsch A 2010 Nat. Mater. 9 868-871

[2] Sakamoto R, Fukui N, Maeda H, Matsuoka R, Toyoda R and Nishihara H 2019 Adv. Mater. 31 1804211

[3] Liu Y, Xue Y, Yu H, Hui L and Huang B andLi Y 2021 Adv. Funct. Mater. 312010112

[4] Zou H, Rong W, Wei S, Ji Y and Duan L 2020 Proc. Natl. Acad. Sci. USA 117 29462-29468

[5] Yu H, Xue Y, Hui L, Zhang C, Fang Y, Liu Y, Chen X, Zhang D, Huang B and Li Y 2021 Natl. Sci. Rev. 88

[6] Baughman R H, Eckhardt H and Kertesz M 1987 J. Chem. Phys. 87 6687-6699

[7] Huang C, Li Y, Wang N, Xue Y, Zuo Z, Liu H and Li Y 2018 Chem. Rev. 118 7744-7803

[8] Casari C S, Tommasini M, Tykwinski R R and Milani A 2016 Nanoscale 8 4414-4435

[9] Casari C S and Milani A 2018 MRS Commun. 8 207-219

[10] Serafini P, Milani A, Proserpio D M and Casari C S 2021 J. Phys. Chem. C 125(33) 18456-18466

[11] Gao X, Liu H, Wang D and Zhang J 2019 Chem. Soc. Rev. 48(3) 908-936

[12] Chen Z, Molina-Jirón C, Klyatskaya S, Klappenberger F and Ruben M 2017 Ann. Phys. 529 1700056

[13] Jia Z, Li Y, Zuo Z, Liu H, Huang C and Li Y 2017 Accounts of Chemical Research 50 2470-2478

[14] Li M, Wang Z K, Kang T, Yang Y, Gao X, Hsu C S, Li Y and Liao L S 2018 Nano Energy 43 $47-54$

[15] Zuo Z, Wang D, Zhang J, Lu F and Li Y 2019 Adv. Mater. 311803762

[16] Li G, Li Y, Liu H, Guo Y, Li Y and Zhu D 2010 Chem. Commun. 46(19) 3256-3258

[17] Li Y, Xu L, Liu H and Li Y 2014 Chem. Soc. Rev. 43 2572-2586

[18] Ivanovskii A L 2013 Progr. Solid State Ch. 41 1-19

[19] Serafini P, Milani A, Tommasini M, Castiglioni C and Casari C S 2020 Phys. Rev. Mater. 4014001

[20] Zhang S, Wang J, Li Z, Zhao R, Tong L, Liu Z, Zhang J and Liu Z 2016 J. Phys. Chem. C 120 10111-10720

[21] Milani A, Tommasini M, Russo V, Bassi A L, Lucotti A, Cataldo F and Casari C S 2015 Beilstein J. Nanotechnol. 6 480-491

[22] Zhang Y Q, Kepčija N, Kleinschrodt M, Diller K, Fischer S, Papageorgiou A C, Allegretti F, Björk J, Klyatskaya S, Klappenberger F, Ruben M and Barth J V 2012 Nat. Commun. 31286

[23] Sun Q, Cai L, Wang S, Widmer R, Ju H, Zhu J, Li L, He Y, Ruffieux P, Fasel R and Xu W 2016 J. Am. Chem. Soc. 138 1106-1109

[24] Sun Q, Tran B V, Cai L, Ma H, Yu X, Yuan C, Stohr M and Xu W 2017 Angew. Chem. Int. Ed. 56 12165-12169

[25] Sun Q, Cai L, Ma H, Yuan C and Xu W 2016 ACS Nano 10 7023-7030

[26] Fan Q, Gottfried J and Zhu J 2015 Acc. Chem. Res. 48 2484-2494

[27] Klappenberger F, Zhang Y Q, Björk J, Klyatskaya S, Ruben M and Barth J V 2015 Acc. Chem. Res. 48 2140-2150 
[28] Shu C H, Liu M X, Zha Z Q, Pan J L, Zhang S Z, Xie Y L, Chen J L, Yuan D W, Qiu X H and Liu P N 2018 Nat. Commun. 92322

[29] Pan Y, Wang Y, Wang L, Zhong H, Quhe R, Ni Z, Ye M, Mei W N, Shi J, Guo W, Yang J and Lu J 2015 Nanoscale 7

[30] Rabia A, Tumino F, Milani A, Russo V, Li Bassi A, Bassi N, Lucotti A, Achilli S, Fratesi G, Onida G, Manini N, Sun Q, Xu W and Casari C S 2020 ACS Appl. Nano Mater. 3 12178-12187

[31] Yan H, Yu P, Han G, Zhang Q, Gu L, Yi Y, Liu H, Li Y and Mao L 2019 Angew. Chem. Int. Ed. $58746-750$

[32] Rabia A, Tumino F, Milani A, Russo V, Li Bassi A, Achilli S, Fratesi G, Onida G, Manini N, Sun Q, Xu W and Casari C S 2019 Nanoscale 11(39) 18191-18200

[33] Borrelli M, Querebillo C, Dominik L P, Tao W, Alberto M, Carlo S C, Ly H K, He F, Hou Y, Christof N, Andrey T, Sun H, Inez M W and Xinliang F 2021 Angew. Chem. Int. Ed. 60 2-8

[34] Galeotti G, Marchi F D, Hamzehpoor E, MacLean O, Rao M R, Chen Y, Besteiro L, Dettmann D, Ferrari L, Frezza F, Sheverdyaeva P, Liu R, Kundu A, Moras P, Ebrahimi M, Gallagher M, Rosei F, Perepichka D and Contini G 2020 Nat. Mater. 19 874-880

[35] Perdew J P, Burke K and Ernzerhof M 1996 Phys. Rev. Lett. 77(18) 3865-3868

[36] Grimme S 2006 J. Comput. Chem. 27(15) 1787-1799

[37] Soler J M, Artacho E, Gale J D, García A, Junquera J, Ordejón P and Sánchez-Portal D $2002 \mathrm{~J}$. Phys.: Condens. Matter 142745

[38] Tersoff J and Hamman D R 1985 Phys. Rev. B 31805

[39] Adamo C and Barone V 1999 J. Chem. Phys. 110 6158-6170

[40] Tommasini M, Fazzi D, Milani A, Del Zoppo M, Castiglioni C and Zerbi G 2007 J. Phys. Chem. A 111 11645-11651

[41] Castelli I, Ferri N, Onida G and Manini N 2012 J. Phys.: Condens. Matter 24104019

[42] Milani A, Barbieri V, Facibeni A, Russo V, Bassi A L, Lucotti A, Tommasini M, Tzirakis M D, Diederich F and Casari C S 2019 Scientific reports 9 1-10

[43] Serafini P, Milani A, Tommasini M, Bottani C E and Casari C S 2021 Carbon 180 265-273

[44] Bryce M R 2021 J. Mater. Chem. C Advance Article() 